\title{
ADJACENT CONNECTED SUMS AND TORUS ACTIONS ${ }^{1}$ \\ BY \\ DENNIS MCGAVRAN
}

\begin{abstract}
Let $M$ and $N$ be closed, compact manifolds of dimension $m$ and let $X$ be a closed manifold of dimension $n<m$ with embeddings of $X \times D^{m-n}$ into $M$ and $N$. Suppose the interior of $X \times D^{m-n}$ is removed from $M$ and $N$ and the resulting manifolds are attached via a homeomorphism $f: X \times S^{m-n-1} \rightarrow X \times S^{m-n-1}$. Let this homeomorphism be of the form $f(x, t)=(x, F(x)(t))$ where $F: X \rightarrow S O(m-n)$. The resulting manifold, written as $M \#_{X} N$, is called the adjacent connected sum of $M$ and $N$ along $X$. In this paper definitions and examples are given and the examples are then used to classify actions of the torus $T^{n}$ on closed, compact, connected, simply connected $(n+2)$-manifolds, $n>4$.
\end{abstract}

Let $M$ and $N$ be closed manifolds of dimension $m$. The connected sum $M \# N$ is obtained by removing the interior of an $m$-cell from each and attaching the resulting manifolds together via a homeomorphism between their boundaries. This construction can be generalized in the following manner. Let $X$ be a closed manifold of dimension $n<m$ and suppose that $X \times D^{m-n}$ is embedded in both $M$ and $N$. Then $\left(X \times D^{m-n}\right)^{\circ}(=$ the interior of $\left.X \times D^{m-n}\right)$ can be removed from each and the resulting manifolds can be joined together via a homeomorphism $f: X \times S^{m-n-1} \rightarrow X \times$ $S^{m-n-1}$ between their boundaries. We shall require that $f$ be of the form $f(x, t)=(x, F(x)(t))$ where $F: X \rightarrow S O(m-n)$. Despite this restriction, the result will not be uniquely determined and will depend upon framings of $X$ in $M$ and $N$. We shall call manifolds obtained in this manner adjacent connected sums.

This construction was used by Goldstein and Lininger in [3] to study free $S^{1}$-actions on simply connected 6-manifolds. If $M$ and $N$ are such manifolds admitting free $S^{1}$-actions, then the interior of an invariant tubular neighborhood of an orbit can be removed from each. These tubular neighborhoods will, of course, be homeomorphic to $S^{1} \times D^{5}$. The resulting manifolds can be attached along $S^{1} \times S^{4}$ to obtain a new simply connected 6-manifold admit-

Received by the editors August 5, 1977.

AMS (MOS) subject classifications (1970). Primary 57E25, 57E10, 57E15, 54B17; Secondary 57C15, 57D05, 57D25.

Key words and phrases. Adjacent connected sums, torus actions, simply connected manifolds, orbit space.

${ }^{1}$ This research was supported in part by a University of Connecticut Faculty Summer Fellowship. 
ting a free $S^{1}$-action. This construction was used to classify all free $S^{1}$-actions on closed, compact, connected, simply connected 6-manifolds with torsion free homology.

This paper was motivated by the realization that similar constructions could be used to study $T^{n}$-actions on simply connected $(n+2)$-manifolds. In $\S \S 1$ and 2 we define adjacent connected sums, determine some of their properties and compute some examples. These examples are then used in $\$ 3$ to classify locally smooth $T^{n}$-actions on closed, compact, connected, simply connected $(n+2)$-manifolds. Such actions were classified by other methods in [13], [14] and [16] for $n=1,2$ and in [11] for $n=3,4$. This paper then completes the classification of all such actions.

Certain notation will be used frequently. $X^{\circ}$ will denote the interior of a space $X . X \cong_{C} Y$ will indicate that $X$ is equivalent to $Y$ in some category $C$ (e.g., PL, equivariant, homotopy). \# ${ }_{k=1}^{n} a_{k} X_{k}$ will denote the connected sum of $a_{k}$ copies of $X_{k}, k=1, \ldots, n . \quad X \cup_{f} Y$ will be the space obtained by attaching $X$ to $Y$ via some homeomorphism $f: A \rightarrow B$ where $A \subset X, B \subset Y$. We will also write this as $X \cup_{A} Y$. In the sphere $S^{n}, S_{+}^{n}$ and $S_{-}^{n}$ will represent the upper and lower hemispheres so that $S_{+}^{n} \cap S_{-}^{n} \simeq S^{n-1}$, the equator. We use $D_{+}^{n}$ and $D_{-}^{n}$ for the upper and lower halves of $D^{n}$.

1. Adjacent connected sums. Let $M$ and $X$ be closed manifolds with $\operatorname{dim} M=m$ and $\operatorname{dim} X=n<m$ and suppose $g: X \times D^{m-n} \rightarrow M$ is an embedding. For convenience identify $(x, y)$ with $g(x, y) \in M$. A smooth framing of $X \times D^{m-n}$ in $M$ is a smooth map $F:\left(X, x_{0}\right) \rightarrow(S O(m-n)$, id). Let $F_{x}$ denote $F(x)$ and let $(M, X, F)$, or simply $(M, F)$ when $X$ is understood, denote $M$ with a particular framing of $X \times D^{m-n}$ in $M$. If $F$ and $G$ are framings we have their composition $F \circ G=H:\left(X, x_{0}\right) \rightarrow(S O(m-$ $n)$, id) where $H_{x}=F_{x} \cdot G_{x}$. The identity framing is $I:\left(X, x_{0}\right) \rightarrow$ (id, id) and we will use $M$ to denote $(M, I)$. By $F^{-1}$ we mean the framing such that $\left(F^{-1}\right)_{x}=\left(F_{x}\right)^{-1}$.

Let $(M, F)$ and $(N, G)$ be framings of $X \times D^{m-n}$ in $M$ and $N$, respectively. The adjacent connected sum, or adjacent sum, of $M$ and $N$ along $X$, denoted by $(M, F) \#_{X}(N, G)$, is obtained in the following manner. Remove from $M$ and $N$ the interiors of the embedded copies of $X \times D^{m-n}$, obtaining $\bar{M}$ and $\bar{N}$, each with boundary homeomorphic to $X \times S^{m-n-1}$. Define $f$ : $\partial \bar{M} \rightarrow \partial \bar{N}$ by $f(x, y)=\left(x, G_{x}^{-1} F_{x}(y)\right)$. Then $(M, F) \#_{X}(N, G)=\bar{M} \cup_{f} \bar{N}$. The following easy lemma shows that we need only consider sums of the form $(M, F) \#_{X} N$.

1.1. Lemma. $(M, F) \#_{X}(N, G) \cong\left(M, G^{-1} \circ F\right) \#_{X} N$.

Let $f: X \times D^{m-n} \rightarrow M$ and $g: X \times D^{m-n} \rightarrow M$ be embeddings and let $(M, F)$ and $(M, G)$ denote framings of their respective images. We say that 
$(M, F)$ is homotopic to $(M, G)$, indicated by $(M, F) \sim(M, G)$, if there is a map $H:\left(X \times D^{m-n} \times I\right) \rightarrow M \times S O(m-n)$ such that $H(x, y, 0)=$ $\left(f(x, y), F_{x}\right)$ and $H(x, y, 1)=\left(g(x, y), G_{x}\right) .(M, F)$ is isotopic to $(M, G)$ if $H(x, y, t)$ is an embedding for each $t$.

In this paper only the identity framing will be used so we will be investigating manifolds of the form $U=M \#_{X} N$. Of course our results will also hold for framings isotopic to the identity.

Now let $M$ and $N$ be $m$-manifolds with boundary and let $D_{M}$ and $D_{N}$ be cells contained in the boundaries of $M$ and $N$ respectively. We will use $M \# N$ to denote $M \cup_{f} N$ where $f: D_{M} \rightarrow D_{N}$ is a homeomorphism. If $X$ is a closed $n$-manifold and we have $X \times D^{m-n-1}$ embedded in $\partial M$ and $\partial N$ then, as before, we can form $(M, F) \#_{X}(N, G)$ where $F$ and $G$ are framings of $X \times D^{m-n-1}$ in $\partial M$ and $\partial N$, respectively.

Suppose $M$ and $N$ are closed manifolds and $\bar{M}$ and $\bar{N}$ are manifolds such that $\partial \bar{M}=M$ and $\partial \bar{N}=N$. It is not difficult to see that $M \#_{X} N=$ $\partial\left(\bar{M} \#_{X} \bar{N}\right)$. This relation is useful since $\bar{M} \#_{X} \bar{N}$ is frequently easier to compute.

2. Examples of $\mathbf{P L}$ adjacent sums. In this section we consider various examples of adjacent connected sums. For the arguments we use, it is necessary to assume that all manifolds and all embeddings are PL.

Although the examples themselves are interesting, they were chosen because of their applications to torus actions in $\$ 3$.

2.1. ExAmple. Suppose $T^{m}$ is embedded via the identity into the second factor of $S^{n} \times T^{m}$. Then $\left(S^{n} \times T^{m}\right) \#_{T^{m}} S^{m+n} \simeq S^{m+n}$. To see this, we proceed as indicated at the end of $\S 1$ and compute $\partial\left[\left(D^{n+1} \times\right.\right.$ $\left.\left.T^{m}\right) \#_{T^{m}} D^{m+n+1}\right]$. We have $D^{n+1} \times T^{m} \simeq I \times\left(D^{n} \times T^{m}\right)$ and we may assume that we are joining this to $D^{n+m+1}$ along $\{0\} \times\left(D^{n} \times T^{m}\right)$. We then obtain $D^{n+m+1}$ so that $\partial\left[\left(D^{n+1} \times T^{m}\right) \#_{T^{m}} D^{m+n+1}\right] \cong S^{n+m}$.

The following is similar to Theorem 3 of [3].

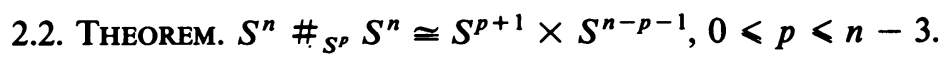

Proof. We consider $D^{n+1} \#_{S^{p}} D^{n+1}$. The (PL) embedded $S^{p}$ in each summand bounds a disk $D^{p+1}$ and we write each $D^{n+1}$ as $D^{p+1} \times D^{n-p}$. Thus we wish to join $D^{p+1} \times D^{n-p}$ to $D^{p+1} \times D^{n-p}$ along $S^{p} \times D^{n-p}$. Since we have the identity framing of $S^{p}$ in each case, we obtain $S^{p+1} \times$ $D^{n-p}$. Therefore $S^{n} \#_{S^{p}} S^{n} \cong S^{p+1} \times S^{n-p-1}$.

The following result and its corollary will be used frequently.

2.3. TheOREM. Let $N=\#_{i=0}^{r} S^{p_{i}} \times D^{n-p_{i}-1}, r>0$, where $n-p_{i}>3$ for each $i$. Let $M$ be an $n$-dimensional manifold with boundary and suppose we have embeddings of $N$ into $\partial M$ and $\partial D^{n}$. Suppose $K=M \cup_{f} D^{n}$ where $f: N \rightarrow N$ is 
induced by the identity framing on each summand $S^{p_{i}} \times D^{n-p_{i}-1}$. Then $K \cong$ $\left(\ldots\left(\left(M \#_{S^{p_{0}}} D^{n}\right) \#_{S^{p_{1}}} \ldots\right) \#_{S^{p_{r}}} D^{n}\right)$.

Proof. We first wish to show that there is a cell $D^{n-1} \subset \partial D^{n}$ such that $D^{n-1} \cap N=S^{p_{0}} \times D^{n-p_{0}-1}$. Let us assume that $N=B \cup\left\{\cup_{i=0}^{r} H_{i}\right\}$ where $B \cong D^{n-1}, H_{i} \cong D^{p_{i}} \times D^{n-p_{i}-1}$ and each $H_{i}$ is attached to $B$ along $S^{p_{i}-1} \times$ $D^{n-p_{i}-1}$, the latter being pairwise disjoint. Also note that $N \approx_{h e}\left(\bigvee_{i=0}^{r} S^{p_{i}}\right)$. Let $X=\partial D^{n}-\left\{\cup_{i=0}^{r} H_{i}^{\circ}\right\}$. Thus $X \cong \# r\left(S^{n-2} \times I\right)$ and $X$ $\cong_{h e}\left\{\bigvee_{j=1}^{r} S^{n-2}\right\}$.

Let $D \subset B^{\circ}$ be an $(n-1)$-cell with $B$ a regular neighborhood of $D$. Let $S^{p_{0}-1} \times I$ be an annulus in $B$ such that $\left(S^{p_{0}-1} \times I\right) \cap \partial B=S^{p_{0}-1} \times\{1\}$ $=\partial D^{p_{0}} \times\left\{x_{0}\right\} \subset \partial H_{0}$ where $x_{0} \in \partial\left(D^{n-p_{0}-1}\right)$, and $\left(S^{p_{0}-1} \times I\right) \cap D=$ $S^{p_{0}-1} \times\{0\} \subset \partial D$. Let $D_{+}^{p_{0}}=\left(D^{p_{0}} \times\left\{x_{0}\right\}\right) \cup\left(S^{p_{0}-1} \times I\right)$, and let $D_{-}^{p_{0}}$ be a $p_{0}$-cell contained in $D^{\circ}$ bounded by $D_{+}^{p_{0}} \cap D=S^{p_{0}-1} \times\{0\}$. Then $S^{p_{0}}=$ $D_{+}^{p_{0}} \cup D_{-}^{p_{0}}$ represents a generator of $\pi_{p_{0}}\left(S^{p_{0}}\right) \subset \pi_{p_{0}}(N) \cong \pi_{p_{0}}\left(\bigvee_{i=0}^{r} S^{p_{i}}\right)$. The embedding $\omega:\left(S^{p_{0}}, S_{-}^{p_{0}}\right) \rightarrow(X, D)$ whose image is $D_{+}^{p_{0}} \cup D_{-}^{p_{0}}$ represents the trivial element of $\pi_{p_{0}}(X, D) \cong \pi_{p_{0}}(X, *) \cong \pi_{p_{0}}\left(\bigvee_{j=1}^{r} S_{j}^{n-2}\right)$, so it is homotopic to an embedding $\sigma:\left(S^{p_{0}}, S_{-}^{p_{0}}\right) \rightarrow(X, D)$ where $\sigma\left(S^{p_{0}}\right)$ bounds a disk in $X-D^{\circ}$. We may in fact assume that $\omega$ is homotopic to $\sigma$ in $\left(X-D^{\circ}, \partial(X\right.$ $\left.\left.-D^{\circ}\right)\right)$. By restriction we have $\omega:\left(S_{+}^{p_{0}}, \partial S_{+}^{p_{0}}\right) \rightarrow\left(X-D^{\circ}, \partial\left(X-D^{\circ}\right)\right)$ homotopic to $\sigma:\left(S_{+}^{p_{0}}, \partial S_{+}^{p_{0}}\right) \rightarrow\left(X-D^{\circ}, \partial\left(X-D^{\circ}\right)\right)$.

Now $\left(S_{+}^{p_{0}}, \partial S_{+}^{p_{0}}\right) \cong_{h e}\left(S^{p_{0}}, *\right)$ is $2 p_{0}-(n-1)+1=2 p_{0}-n+2$ connected since $n \geqslant p_{0}+3 .\left(X-D^{\circ}, \partial\left(X-D^{\circ}\right)\right) \cong\left(\#(r+1)\left(S^{n-2} \times I\right)\right.$, $\left.\partial\left(\#(r+1)\left(S^{n-2} \times I\right)\right)\right)$ so it is $2 p_{0}-(n-1)+2=2 p_{0}-n+3$ connected. Therefore, by 10.2 of [5], we have $\omega$ isotopic to $\sigma$.

Thus we may assume that $S^{p_{0}}=D_{+}^{p_{0}} \cup D_{-}^{p_{0}}$ bounds a disk in $X-D^{\circ}$. Let $\bar{D}_{0}^{n-1}$ be the normal bundle of this disk with $H_{0} \cup \bar{D}_{0}^{n-1} \cong D^{n-1}$. Choose a regular neighborhood $D_{0}^{n-1}$ of $H_{0} \cup \bar{D}_{0}^{n-1}$ such that $D_{0}^{n-1} \cap N=S^{p_{0}} \times$ $D^{n-p_{0}-1}$ and let $D_{0}^{n}$ be a collared neighborhood of $D_{0}^{n-1}$. Remove $D_{0}^{n}$ from $D^{n}$ and form $M_{0}=M \#_{S^{p_{0}}} D_{0}^{n}$. We then need $M_{0} \cup_{Y}\left(D^{n}-\left(D_{0}^{n}\right)^{\circ}\right)$ where $Y=\left(N-\left(S^{p_{0}} \times D^{n-p_{0}-1}\right)^{\circ}\right) \cup_{D^{n-2}} D^{n-1}$. Thus $Y \cong \#_{i=1}^{r} S^{p_{i}} \times D^{n-p_{i}-1}$ and we want $\left(M \#_{S^{p_{0}}} D_{0}^{n}\right) \cup_{Y} D^{n}$. Continuing in this manner proves the theorem.

2.4. Corollary. With the same hypotheses as in 2.3 , suppose $M \cong D^{n}$. Then $K \cong \#_{i=0}^{r} S^{p_{i}+1} \times D^{n-p_{i}-1}$.

Proof. We want $K=\left(D_{1}^{n}\right) \cup \cup_{N}\left(D_{2}^{n}\right), N \cong \#_{i=0}^{r} S^{p_{i}} \times D^{n-p_{i}-1}$. As in the proof of 2.3 , find $D_{01}^{n}$ such that $D_{1}^{n}=\left(D_{1}^{n}-\left(D_{01}^{n}\right)^{\circ}\right) \cup D_{01}^{n},\left(D_{1}^{n}-\left(D_{01}^{n}\right)^{\circ}\right) \cap$ $D_{01}^{n} \cong D_{1}^{n-1}, S^{p_{0}} \times D^{n-p_{0}-1} \subset \partial D_{01}^{n}$ and

$$
\left(\left(\partial D_{1}^{n}\right) \cap D_{01}^{n}\right) \cap\left(N-\left(\#_{i=1}^{r} S^{p_{i}} \times D^{n-p_{i}-1}\right)^{\circ}\right) \simeq D^{n-2} \subset D_{1}^{n-1}
$$


Likewise find $D_{02}^{n} \subset D_{2}^{n}$ satisfying corresponding properties. Contained in $K$ we have $X=D_{01}^{n} \#_{S^{p_{0}}} D_{02}^{n} \cong S^{p_{0}+1} \times D^{n-p_{0}-1}$. Furthermore, $X$ will be attached to $K-X^{\circ}$ along $D_{1}^{n-1} \cup_{D^{n-2}} D_{2}^{n-1} \simeq D^{n-1}$. Thus $K \cong(K-$ $\left.X^{\circ}\right) \# S^{p_{0}+1} \times D^{n-p_{0}-1}$. Continuing in this manner proves the corollary.

We now wish to consider $S^{n-1} \#_{S^{p} \times T^{k}}\left(S^{n-k-1} \times T^{k}\right)$ where the embed$\operatorname{ding} S^{p} \times T^{k} \rightarrow S^{n-k-1} \times T^{k}$ is a product of an embedding $S^{p} \rightarrow S^{n-k-1}$ and a homeomorphism $T^{k} \rightarrow T^{k}$. We first prove the following lemma.

2.5. LEMMA. Suppose $0 \leqslant p \leqslant n-5$ and suppose we have an embedding $S^{p} \times D^{n-p-2} \times T^{1} \rightarrow \partial\left(D^{n-1} \times T^{1}\right)$ which is the product of an embedding $S^{p} \times D^{n-p-2} \rightarrow \partial D^{n-1}$ and a homeomorphism $T^{1} \rightarrow T^{1}$. Suppose we have $D^{n}=D^{p+1} \times D^{n-p-1}$ and an embedding $S^{p} \times T^{1} \times D^{n-p-2} \rightarrow D^{n}$ which is the product of $S^{p} \rightarrow \partial D^{p+1}$ and an embedding $T^{1} \times D^{n-p-2} \rightarrow D^{n-p-1}$. With these embeddings,

$$
D^{n} \#_{S^{p} \times T^{1}} D^{n-1} \times T^{1} \cong S^{p+1} \times D^{n-p-1} \# S^{p+2} \times D^{n-p-2} .
$$

Proof. We consider $D^{n-1} \times T^{1}$ as $\left(D^{n-1} \times I\right)_{1} \#_{S^{0}}\left(D^{n-1} \times I\right)_{2}$. We first find

$$
M_{1}=D^{n} \#_{S^{p} \times I}\left(D^{n-1} \times I\right)_{1} \cong D^{n} \#_{S^{p}} D^{n} \cong S^{p+1} \times D^{n-p-1} .
$$

We now have that

$$
M=D^{n} \#_{S^{p} \times T^{1}} D^{n-1} \times T^{1} \cong M_{1} \cup_{X}\left(D^{n-1} \times I\right)_{2}
$$

where $X=S^{p} \times D^{n-p-2} \times I \cup D^{n-1} \times S^{0}$ and these are joined along $S^{p}$ $\times D^{n-p-2} \times S^{0}$. It is not difficult to see that $X \cong S^{p+1} \times D^{n-p-2}$ where $D^{n-1} \times S^{0}$ is the union of the north and south polar caps. Thus we want $M \cong M_{1} \#_{S^{p+1}}\left(D^{n-1} \times I\right)_{2}$.

We need only show that $X$ is contained in an $(n-1)$-cell in $\partial M_{1}$. It will then follow that

$$
M \cong M_{1} \#\left(D^{n} \#_{S^{p+1}} D^{n}\right) \cong S^{p+1} \times D^{n-p-1} \# S^{p+2} \times D^{n-p-2} .
$$

First write $D^{n-1}$ as $D^{p+1} \times D^{n-p-2}$ where the image of the embedding $S^{p} \times D^{n-p-2} \rightarrow D^{n-1}$ is $\partial\left(D^{p+1}\right) \times D^{n-p-2}$. In $\left(D^{n-1} \times I\right)_{1}$ we have $\left(D^{p+1} \times D^{n-p-3}\right) \times I \cong D^{n-2} \times I$ and joining this (in $\left.\left(D^{n-1} \times I\right)_{1}\right)$ to $D^{n-1} \times S^{0}$ along $D^{n-2} \times S^{0}$ gives $D^{n-1}=Y$. Now we have $D^{n}=D^{p+1} \times$ $D^{2} \times D^{n-p-3}$ where the image of $S^{p} \times T^{1} \times D^{n-p-2} \rightarrow D^{n}$ is $\partial\left(D^{p+1}\right) \times$ $\left(\partial D^{2} \times I\right) \times D^{n-p-3}$. We may join, in $\partial D^{n}, S^{p} \times D^{2} \times D^{n-p-3}$ to $S^{p} \times(I$ $\times I) \times D^{n-p-3}$ along $S^{p} \times I \times D^{n-p-3}$ and still have $S^{p} \times D^{2} \times D^{n-p-3}$. But now we can take this $S^{p} \times D^{2} \times D^{n-p-3}$ and join $Y$ to it along $S^{p} \times I \times D^{n-p-3}$ and get a cell $D^{n-1}$ containing $X$.

2.6. Corollary. With the embeddings in $2.5, S^{n-1} \#_{S^{p} \times T^{1}} S^{n-2} \times T^{1} \simeq$ $S^{p+1} \times S^{n-p-2} \# S^{p+2} \times S^{n-p-3}$. 
Before we generalize this result we need to make some observations about tori embedded in Euclidean space. We may embed $T^{2}$ in $\mathbf{R}^{3}$ in the following manner. Take the unit circle in the $(x, y)$-plane, translate it via the vector $(0,2,0)$ and then rotate about the $x$-axis. Inductively, suppose we have $T^{k-1} \subset \mathbf{R}^{k} \subset \mathbf{R}^{k+1}$ with center at the origin. Translate this 2 units along the $x_{k}$-axis and rotate in the $\left(x_{k}, x_{k+1}\right)$-plane. In terms of coordinates, we have

$$
\begin{array}{r}
T^{k}=\left\{\left(\cos \alpha_{1}, \ldots, \cos \alpha_{i}\left(2+\sin \alpha_{i-1}\left(2+\sin \alpha_{i-2}\left(\ldots\left(2+\sin \alpha_{1}\right) \ldots\right)\right)\right),\right.\right. \\
\ldots, \cos \alpha_{k}\left(2+\sin \alpha_{k-1}(\ldots)\right), \\
\left.\left.\sin \alpha_{k}\left(2+\sin \alpha_{k-1}(\ldots)\right)\right) \mid 0<\alpha_{i}<2 \pi\right\} .
\end{array}
$$

We shall call this the standard torus $T^{k} \subset \mathbf{R}^{k+1}$. Let $\alpha=\left(\alpha_{1}, \ldots, \alpha_{k}\right)$ and consider the isotopy $F: T^{k} \times I \rightarrow D^{k+2}$ defined by

$$
\begin{array}{r}
F(\alpha, t)=\left(\cos \alpha_{1}, \ldots, \cos \alpha_{i}\left(2+\sin \alpha_{i-1}\left(\ldots\left(1+t+t \sin \alpha_{1}\right) \ldots\right)\right),\right. \\
\left.\ldots, \sin \alpha_{k}\left(2+\sin \alpha_{k-1}(\ldots)\right),(1-t) \sin \alpha_{1}\right) .
\end{array}
$$

An examination of this isotopy proves the following.

2.7. LEMma. Let $T^{k}$ be the standard torus in $D^{k+1} \subset D^{n}, n>k+1$. Then we may write $D^{n}$ as $D^{2} \times D^{n-2}$ so that $T^{k}$ is isotopic to $\left(\partial D^{2}\right) \times T^{k-1}$ where $T^{k-1}$ is a standard torus in $D^{k} \subset D^{n-2}$.

We shall call an embedding as described in Lemma 2.7 the standard torus in $\partial D^{n} \cong S^{n-1}$.

By modifying $F$ slightly we have the following.

2.8. LEMMA. Suppose we have an embedding $f:\left(T^{k} \times I\right) \times D^{n-k-1} \rightarrow D^{k+1}$ $\times D^{n-k-1}=D^{n}$ which is the product of the identity $D^{n-k-1} \rightarrow D^{n-k-1}$ and an embedding $T^{k} \times I \rightarrow D^{k+1}$ whose image is a product neighborhood of a standard $T^{k} \subset D^{k+1}$. We may write $D^{n}$ as $D^{2} \times D^{k} \times D^{n-k-2}$ so that $f$ is isotopic to an embedding whose image is $\left(\partial D^{2} \times I\right) \times\left(T^{k-1} \times I\right) \times D^{n-k-2}$ where $T^{k-1} \times I$ is a product neighborhood of a standard $T^{k-1} \subset D^{k}$.

For our applications to adjacent connected sums we need to have another isotopy that modifies a standard $T^{k} \times D^{n-k-1} \subset D^{k+1} \times D^{n-k-1}$. To this end we consider

$$
T^{k} \times I \times D^{n-k-2}=\left\{(\alpha, z) \mid \alpha \in T^{k} \subset D^{k+1},-1<z<1\right\} \times D^{n-k-2} \text {. }
$$

Define

$$
\begin{aligned}
G((\alpha, z), t)=( & \cos \alpha_{1}, \frac{1}{4}((z-1)(1-t)+4) \cos \alpha_{2}\left(1+t+t \sin \alpha_{1}\right) \\
& \ldots, \frac{1}{4}((z-1)(1-t)+4) \\
& \cdot \cos \alpha_{i}\left(2+\sin \alpha_{i-1}\left(\ldots\left(1+t+t \sin \alpha_{1}\right) \ldots\right)\right) \\
& \ldots, \frac{1}{4}((z-1)(1-t)+4) \sin \alpha_{k}\left(2+\sin \alpha_{k-1}(\ldots) \ldots\right) \\
& \left.(1-t) \sin \alpha_{1}+z t\right)
\end{aligned}
$$


Examination of this proves the following.

2.9. Lemma. Suppose $k<n-2$. Suppose $f: T^{k} \times D^{n-k-1} \rightarrow D^{k+1} \times$ $D^{n-k-1}$ is the product of a standard embedding of $T^{k} \rightarrow D^{k+1}$ and the identity $D^{n-k-1} \rightarrow D^{n-k-1}$. Then we can write $D^{n}$ as $D^{2} \times D^{k} \times D^{n-k-2}$ and $f$ is isotopic to an embedding $g$ whose image is $\partial D^{2} \times\left(T^{k-1} \times I\right) \times D^{n-k-2}$ where $T^{k-1} \times I$ is a product neighborhood of a standard $T^{k-1} \subset D^{k}$.

2.10. THEOREM. Suppose $0<p<n-k-4$. Let $S^{p} \times D^{n-p-k-1} \times T^{k}$ $\rightarrow S^{n-k-1} \times T^{k}$ be a product of an embedding $S^{p} \times D^{n-p-k-1} \rightarrow S^{n-k-1}$ and a homeomorphism $T^{k} \rightarrow T^{k}$. Let $S^{n-1}=\partial D^{n}=\partial\left(D^{p+1} \times D^{n-p-1}\right)$ and let $S^{p} \times D^{n-p-k-1} \times T^{k}$ be a product of the inclusion $S^{p} \rightarrow \partial D^{p+1}$ and an embedding $\left(T^{k} \times I\right) \times D^{n-p-k-2} \rightarrow D^{k+1} \times D^{n-p-k-2}$, where the image of $T^{k} \times I$ is a product neighborhood of a standard $T^{k} \subset D^{k+1}$. With these embeddings

$$
\left(S^{n-k-1} \times T^{k}\right) \#_{S^{p} \times T^{k}} S^{n-1} \cong \#_{j=0}^{k}\left(\begin{array}{l}
k \\
j
\end{array}\right) S^{p+1+j} \times S^{n-p-j-2} .
$$

Proof. By 2.6, the theorem is true for $k=1$ so suppose it is true for $K-1$ and consider $M=D^{n-K} \times T^{K} \#_{S^{p} \times T^{K}} D^{n}$. Let $T^{K}=T_{1} \times T^{K-1}$. By 2.8, we may assume that $D^{n}=D^{p+1} \times D^{2} \times D^{K} \times D^{n-p-K-3}$ and our embedding $S^{p} \times D^{n-p-K-1} \times T^{K} \rightarrow D^{n}$ is a product of the inclusions $S^{p}$ $\rightarrow \partial D^{p+1},\left(T_{1} \times I\right) \rightarrow\left(\partial D^{2} \times I\right)$, the identity $D^{n-p-K-3} \rightarrow D^{n-p-K-3}$ and an embedding $\left(T^{K-1} \times I\right) \rightarrow D^{K}$ whose image is a product neighborhood of a standard $T^{K-1} \subset D^{K}$. First we have

$$
\begin{aligned}
M_{1} & =D^{n-K} \times I \times T^{K-1} \#_{S^{p} \times I \times T^{K-1}} D^{n} \\
& \cong D^{n-K+1} \times T^{K-1} \#_{S^{p} \times T^{K-1}} D^{n} \\
& =\#_{j=0}^{K-1}\left(\begin{array}{c}
K-1 \\
j
\end{array}\right) S^{p+1+j} \times D^{n-p-j-1}
\end{aligned}
$$

Then $M=M_{1} \cup_{X} D^{n-K} \times I \times T^{K-1}$ where

$$
X=D^{n-K} \times S^{0} \times T^{K-1} \cup_{Y} S^{p} \times D^{n-K-p-1} \times I \times T^{K-1}=X_{1} \cup_{Y} X_{2}
$$

where $Y=S^{p} \times D^{n-K-p-1} \times S^{0} \times T^{K-1}$. Thus

$$
\begin{aligned}
X & \cong D^{n-K-p-1} \times T^{K-1} \times\left(D^{p+1} \times S^{0} \cup_{S^{p} \times S^{0}} S^{p} \times I\right) \\
& \simeq D^{n-K-p-1} \times T^{K-1} \times S^{p+1} .
\end{aligned}
$$

Therefore $M=M_{1} \#_{S^{p+1} \times T^{K-1}} D^{n-K+1} \times T^{K-1}$.

As before we wish to show that $X$ is contained in an $(n-1)$-cell in $\partial M_{1}$. In $\partial\left(D^{n-K} \times I \times T^{K-1}\right)$ we have $D^{p+1} \times D^{n-K-p-2} \times I \times T^{K-1}$ which we may join to $X_{1}$ along $D^{n-K-1} \times S^{0} \times T^{K-1}$ to get $D^{n-K} \times T^{K-1}=W_{1}$. In $\partial D^{n}$ we have $S^{p} \times D^{n-K-p-2} \times D^{2} \times T^{K-1}$, where $\partial D^{2}$ corresponds to $T_{1}$, 
and we may join this to $X_{2}$ along $S^{p} \times D^{n-K-p-2} \times I \times T^{K-1}$ to get $S^{p} \times D^{n-K-p-2} \times D^{2} \times T^{K-1}=W_{2}$. Thus we have $X=X_{1} \cup_{Y} X_{2} \subset W_{1}$ $\cup_{Z} W_{2} \cong D^{n-K} \times T^{K-1} \subset \partial M_{1}$ where $Z \cong S^{p} \times D^{n-K-p-2} \times I \times T^{K-1}$. Contained in this copy of $D^{n-K} \times T^{K-1}$ we have $U_{1}=S_{+}^{p} \times D^{n-K-p-2} \times$ $D^{2} \times T^{K-1} \subset W_{2}$. Thus $U_{1} \cong D^{n-K} \times T^{K-1}$. In fact we may assume $D^{n-K}$ $\times T^{K-1}=U_{1} \cup_{V_{1}}\left(\left(D^{n-K} \times T^{K-1}\right)-U_{1}^{\circ}\right)$ where $V_{1}=S_{+}^{p} \times D^{n-K-p-2} \times I \times T^{K-1} \cup_{V_{2}} S^{p-1} \times D^{n-K-p-2} \times D^{2} \times T^{K-1}$, $V_{2}=S^{p-1} \times D^{n-K-p-2} \times I \times T^{K-1}$.

Then $V_{1} \cong D^{n-K-1} \times T^{K-1}$. Therefore $D^{n-K} \times T^{K-1}=U_{1}$ $\cup_{D^{n-K-1} \times T^{K-1}}\left(D^{n-K} \times T^{K-1}\right)$. Now

$$
U_{1} \subset S_{+}^{p} \times D^{2} \times D^{K} \times D^{n-p-K-3}=U_{2} \simeq D^{n-1} .
$$

Thus $D^{n-K} \times T^{K-1} \subset U_{2} \cup_{V_{1}}\left(D^{n-K} \times T^{K-1}\right) \cong D^{n-1}$. Furthermore the embedding of $X$ into $D^{n-1}$ will be of the type described in this theorem.

It follows then that

$$
\begin{aligned}
M \cong & M_{1} \#\left(D^{n} \#_{S^{p+1} \times T^{K-1}} D^{n-K+1} \times T^{K-1}\right) \\
\cong & M_{1} \#\left(\begin{array}{c}
K-1 \\
\#_{j=0}
\end{array}\left(\begin{array}{c}
K-1 \\
j
\end{array}\right) S^{p+2+j} \times D^{n-p-j-2}\right) \\
\simeq & \left(\begin{array}{c}
K-1 \\
j=0
\end{array}\left(\begin{array}{c}
K-1 \\
j
\end{array}\right) S^{p+1+j} \times D^{n-p-j-1}\right) \\
& \#\left(\begin{array}{c}
K-1 \\
j=0
\end{array}\left(\begin{array}{c}
K-1 \\
j
\end{array}\right) S^{p+2+j} \times D^{n-p-j-2}\right) \\
= & S^{p+1} \times D^{n-p-1} \#\left(\begin{array}{c}
K-1 \\
j=1
\end{array}\left(\begin{array}{c}
K-1 \\
j
\end{array}\right) S^{p+1+j} \times D^{n-p-j-1}\right) \\
& \#\left(\begin{array}{c}
K-1 \\
j=1
\end{array}\left(\begin{array}{c}
K-1 \\
j-1
\end{array}\right) S^{p+1+j} \times D^{n-p-j-1}\right) \# S^{p+K+1} \times D^{n-p-K-1} \\
= & \#\left(\begin{array}{c}
K \\
j
\end{array}\right) S^{p+1+j} \times D^{n-p-j-1} .
\end{aligned}
$$

Thus

$$
\left(S^{n-K-1} \times T^{K}\right) \#_{S^{p} \times T^{K}} S^{n-1} \cong \partial M \cong \#_{j=0}^{K}\left(\begin{array}{c}
K \\
j
\end{array}\right) S^{p+1+j} \times S^{n-p-j-2} .
$$

We now wish to consider $S^{n-1} \#_{T^{k}} S^{n-1}$. We start with the following. 
2.11. LemMA. Suppose we have regular embeddings of $T^{n-4}$ into the boundaries of two copies of $D^{n}, n>5$. Then

$$
D^{n} \#_{T^{n-4}} D^{n}=\#_{j=1}^{n-4}\left(\begin{array}{c}
n-4 \\
j
\end{array}\right) S^{j+1} \times D^{n-j-1} \text {. }
$$

Proof. This is true for $n=5$ by 2.2. Therefore we use induction and consider $D^{n} \#_{T^{n-4}} D^{n}$. We may assume that $D^{n}=D^{2} \times D^{n-4} \times D^{2}$ and $T^{n-4} \times D^{3}=T_{1} \times T^{n-5} \times I \times D^{2}=\partial D^{2} \times\left(T^{n-5} \times I\right) \times D^{2}$ where $T^{n-5} \times I$ is regularly embedded in $D^{n-4}$. Let $S_{+}^{1}$ and $S_{-}^{1}$ be the upper and lower hemispheres of $T_{1}$. We first form $M_{1}=D^{n} \cup_{Y} D_{+}^{2} \times D^{n-2}, Y=S_{+}^{1}$ $\times T^{n-5} \times D^{3}$.We have

$$
\begin{aligned}
M_{1} & \simeq I \times D^{n-1} \cup_{Y} I \times D^{n-1}=I \times\left(\begin{array}{c}
n-5 \\
\prod_{j=1}
\end{array}\left(\begin{array}{c}
n-5 \\
j
\end{array}\right) S^{j+1} \times D^{n-j-2}\right) \\
& \simeq \#_{j=1}^{n-5}\left(\begin{array}{c}
n-5 \\
j
\end{array}\right) S^{j+1} \times D^{n-j-1} .
\end{aligned}
$$

We now need $M=M_{1} \cup_{X}\left(D_{-}^{2} \times D^{n-2}\right)$ where

$$
\begin{aligned}
& X=I \times D^{n-2} \cup_{Z} S_{-}^{1} \times T^{n-5} \times D^{3} \cong D^{n-1} \#_{S^{0} \times T^{n-5}} D^{4} \times T^{n-5} \\
& Z=S^{0} \times T^{n-5} \times D^{3} .
\end{aligned}
$$

By $2.10, X \simeq \#_{j=0}^{n-5}\left(\begin{array}{c}n-5 \\ j\end{array}\right) S^{1+j} \times D^{n-2-j}$. As usual we wish to show that $X$ is contained in an $(n-1)$-cell $D^{n-1} \subset \partial M_{1}$. We write

$$
X=I \times D^{n-4} \times I \times I \cup_{Z} S_{-}^{1} \times\left(T^{n-5} \times I\right) \times I \times I .
$$

In $D_{+}^{2} \times D^{n-2}$ we consider

$$
\begin{gathered}
I \times D^{n-2} \subset U_{1}=D_{+}^{2} \times T^{n-5} \times I \times I \times\{1\} \cup_{V_{1}} I \times D^{n-4} \times I \times I \\
\simeq D^{n-1} \subset \partial\left(D_{+}^{2} \times D^{n-2}\right) \\
V_{1}=I \times T^{n-5} \times I \times I \times\{1\}
\end{gathered}
$$

Furthermore, in $M_{1}$,

$$
\begin{aligned}
W=U_{1} \cap \partial D^{n} \cong & \left(S_{+}^{1} \times T^{n-5} \times I \times I \times\{1\}\right) \\
& U_{W_{1}}\left(S^{0} \times T^{n-5} \times I \times I \times I\right) \\
\cong & I \times T^{n-5} \times D^{2}, \\
& W_{1} \cong S^{0} \times T^{n-5} \times\{1\} \times D^{2} .
\end{aligned}
$$


In $D^{n}$ we have

$$
\begin{aligned}
S_{-}^{1} \times T^{n-5} \times D^{3} \subset U_{2}= & S_{-}^{1} \times T^{n-5} \times I \times I \times I \\
& U_{V_{2}} D^{2} \times T^{n-5} \times I \times I \times\{1\} \\
\simeq & D^{2} \times T^{n-5} \times D^{2}
\end{aligned}
$$

where

$$
V_{2}=S_{-}^{1} \times T^{n-5} \times I \times I \times\{1\}
$$

In $M_{1}$,

$$
\begin{aligned}
U_{2} \cap \partial\left(D_{+}^{2} \times D^{n-2}\right) \simeq & \left(S^{0} \times T^{n-5} \times I \times I \times I\right) \\
& U_{W_{2}}\left(S_{+}^{1} \times T^{n-5} \times I \times I \times\{1\}\right) \\
\simeq & I \times T^{n-5} \times D^{2}=W
\end{aligned}
$$

where

$$
W_{2}=S^{0} \times T^{n-5} \times I \times I \times\{1\} .
$$

Thus we have $X \subset U_{1} \cup_{W} U_{2} \simeq D^{n-1} \subset \partial M_{1}$.

As before we get $M=M_{1} \#\left(D^{n} \cup_{X} D^{n}\right)$. By 2.4,

$$
\begin{aligned}
M & \simeq M_{1} \#\left(\begin{array}{c}
n-5 \\
j=0
\end{array}\left(\begin{array}{c}
n-5 \\
j
\end{array}\right) S^{2+j} \times D^{n-2-j}\right) \\
& =\left(\begin{array}{c}
n-5 \\
j=1
\end{array}\left(\begin{array}{c}
n-5 \\
j
\end{array}\right) S^{j+1} \times D^{n-j-1}\right) \#\left(\begin{array}{c}
n-1 \\
j=1
\end{array}\left(\begin{array}{c}
n-5 \\
j-1
\end{array}\right) S^{1+j} \times D^{n-j-1}\right) \\
& \# S^{n-3} \times D^{3} \\
& =\underset{j=1}{n-4}\left(\begin{array}{c}
n-4 \\
j
\end{array}\right) S^{j+1} \times D^{n-j-1} .
\end{aligned}
$$

2.12. THEOREM. Suppose we have standard embeddings of $T^{k} \rightarrow S^{n-1}, n-k$ 4. Then

$$
S^{n-1} \#_{T^{k}} S^{n-1} \simeq \#_{j=1}^{k}\left(\begin{array}{l}
k \\
j
\end{array}\right) S^{j+1} \times S^{n-j-2}
$$

Proof. We first consider $D^{n} \#_{T^{k}} D^{n}$, i.e., if we let $X=T^{k} \times D^{3} \times$ $D^{n-k-4}$, we want 


$$
\begin{aligned}
D^{n} \cup_{X} D^{n} & =D^{k+4} \times D^{n-k-4} \cup_{X} D^{k+4} \times D^{n-k-4} \\
& \simeq\left(D^{k+4} \# T^{k} D^{k+4}\right) \times D^{n-k-4} \\
& \left.=\left(\begin{array}{c}
k \\
j=1 \\
j
\end{array}\right) S^{j+1} \times D^{k-j+3}\right) \times D^{n-k-4} \\
& =\#_{j=1}^{k}\left(\begin{array}{l}
k \\
j
\end{array}\right) S^{j+1} \times D^{n-j-1} .
\end{aligned}
$$

It follows that

$$
S^{n-1} \#_{T^{k}} S^{n-1} \cong \#_{j=1}^{k}\left(\begin{array}{c}
k \\
j
\end{array}\right) S^{j+1} \times S^{n-j-2} .
$$

We now prove the following lemma which is essential to our classification theorems in the next section.

2.13. LEMMA. Let $T^{n-4}=T_{1} \times T^{n-5}$. Suppose we have product embeddings $T_{1} \times\left(T^{n-5} \times D^{4}\right) \rightarrow S^{1} \times D^{n}$ and $\left(T_{1} \times D^{4}\right) \times T^{n-5} \rightarrow D^{6} \times T^{n-5}$ (where $T^{n-5} \rightarrow D^{n}$ is regular). With these embeddings

$$
D^{6} \times T^{n-5} \#_{T^{n-4}} S^{1} \times D^{n} \simeq \#_{j=1}^{n-5}\left(\begin{array}{c}
n-5 \\
j
\end{array}\right) S^{j+2} \times D^{n-j-1}
$$

Proof. We first form $M_{1}=T^{n-5} \times D^{6} \cup_{X} I \times D^{n} \simeq I \times D^{n}$, where $X=I \times T^{n-5} \times D^{4}$. Then we form $M=I \times D^{n} \cup_{Y} I \times D^{n}$ where $Y=$ $S^{0} \times D^{n} \cup_{S^{0} \times Z} I \times T^{n-5} \times D^{4}, Z=T^{n-5} \times D^{4}$. Let $I^{+}=\{x \in I=$ $[-1,1] \mid x>0\}, I^{-}=\{x \in I \mid x<0\}$. Then $I \times T^{n-5} \times D^{4}=\left(I^{+} \times\right.$ $\left.T^{n-5} \times D^{4}\right) \#_{T^{n-5}}\left(I^{-} \times T^{n-5} \times D^{4}\right)$. To find $Y$ we first form $\left(I^{+} \times T^{n-5}\right.$ $\left.\times D^{4}\right) \cup_{Z} D^{n} \cong D^{n}$ and $\left(I^{-} \times T^{n-5} \times D^{4}\right) \cup_{Z} D^{n} \cong D^{n}$ so that

$$
Y \cong D^{n} \#_{T^{n-s}} D^{n} \cong \#_{j=1}^{n-5}\left(\begin{array}{c}
n-5 \\
j
\end{array}\right) S^{j+1} \times D^{n-j-1} \text {. }
$$

Then

$$
M \cong D^{n+1} \cup_{Y} D^{n+1} \cong \#_{j=1}^{n-5}\left(\begin{array}{c}
n-5 \\
j
\end{array}\right) S^{j+2} \times D^{n-j-1} .
$$

3. Torus actions. Let $M^{n+2}$ be a closed, compact, connected, simply connected $(n+2)$-manifold and suppose the torus $T^{n}$ acts on $M^{n+2}$ in a locally smooth manner. In this section we classify all such actions for $n>4$. By this we mean that we will be able to identify the manifold if we know what the weighted orbit space looks like. Such actions were classified for $n=1,2$ in [13], [14] and [16] and for $n=3,4$ in [11]. Thus, with these results, such actions are classified for all $n$. Unless stated differently all manifolds are assumed to be compact and connected and all actions are assumed to be locally smooth and effective. 
Now suppose that $T^{n}$ acts on a closed, simply connected $(n+2)$-manifold $M$. In [6] the following results were established: (1) the orbit space $M^{*}$ is a disk $D^{2}$; (2) all stability (isotropy) groups are isomorphic to $T^{1}=S^{1}$ or $T^{2}=S^{1} \times S^{1}$; (3) the interior points of $M^{*}$ correspond to principal orbits; (4) isolated points on $\partial\left(M^{*}\right)$ correspond to orbits of type $T^{n-2}$ (isotropy type $\left.T^{2}\right)$ while the remaining points on $\partial\left(M^{*}\right)$ correspond to orbits of type $T^{n-1}$; (5) the $T^{1}$-stability groups span $T^{\mathrm{n}}$; (6) cross-sections exist; (7) such actions exist for all $n$. The existence of cross-sections was used to prove an equivariant classification theorem, i.e., actions with identical weighted orbit spaces are equivariantly homeomorphic. The last result was significant since no such actions were known for $n>4$. In [11] it was shown that there were $T^{1}$-stability groups $T_{1}, \ldots, T_{n}$ such that $T^{n} \cong T_{1} \cdots \times T_{n}$. It was also shown that for each $n$, there is a unique simply connected manifold $M_{n+2}$ admitting an action of $T^{n}$ satisfying the following condition: there are exactly $n T^{1}$-stability groups $T_{1}, \ldots, T_{n}$ with each $F\left(T_{i}, M_{n+2}\right)$ connected. It was shown that these manifolds, $M_{n+2}$, serve as building blocks for the construction of other actions in the following manner. If $\left(T^{n}, M^{n+2}\right), \pi_{1}\left(M^{n+2}\right) \cong 0$, is any action, then it can be obtained from an action $\left(T^{n}, M_{n+2}\right)$ by equivariantly replacing copies of $D^{4} \times T^{n-2}$ with copies of $S^{3} \times D^{2} \times T^{n-3}$. The following diagram shows how this replacement occurs. It can easily be seen that $M_{5}=S^{5}$ and $M_{6}=S^{3} \times S^{3}$. In this section we first identify all the $M_{n+2}$. We then classify all actions $\left(T^{n}, M^{n+2}\right)$.

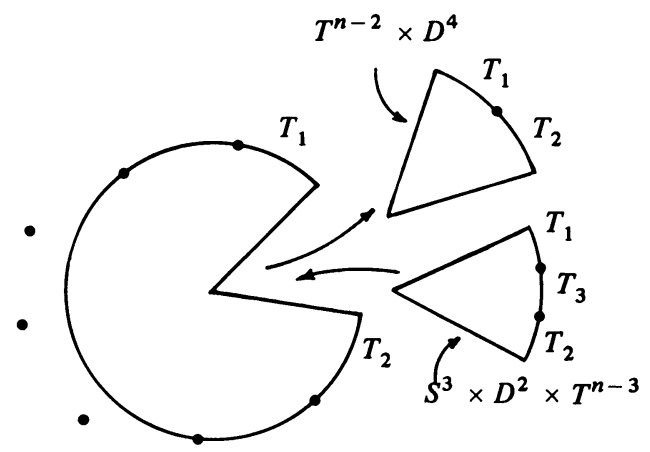

We are considering locally smooth actions while in the last section we worked entirely in the PL-category. We now give some lemmas that will enable us to apply the PL-examples to the actions of this section. We first need some terminology. We shall say that $\left(T_{1}, T_{2}\right)$ is an adjacent pair of $T^{1}$-stability groups for an action $\left(T^{n}, M^{n+2}\right)$ if there is an invariant $D^{4} \times$ $T^{n-2}$ so that the induced action $\left(T^{n}, D^{4} \times T^{n-2}\right)$ has stability groups $T_{1}, T_{2}$ and $T_{1} \times T_{2}$. $\left(T_{1}, T_{2}, T_{3}\right)$ will be called an adjacent triple of $T^{1}$-stability 
groups if $\left(T_{1}, T_{2}\right)$ and $\left(T_{2}, T_{3}\right)$ are adjacent pairs, with invariant copies of $D^{4} \times T^{n-2},\left(D^{4} \times T^{n-2}\right)_{1}$ and $\left(D^{4} \times T^{n-2}\right)_{2}$, respectively, such that $\left(D^{4} \times\right.$ $\left.T^{n-2}\right)_{1} \cap\left(D^{4} \times T^{n-2}\right)_{2} \cong D^{2} \times T^{n-1}$ and $\{0\} \times T^{n-1} \subset F\left(T_{2}, M^{n+2}\right)$. In this case we say that $\left(D^{4} \times T^{n-2}\right)_{1}$ and $\left(D^{4} \times T^{n-2}\right)_{2}$ are adjacent and we let $X\left(T_{1}, T_{2}, T_{3}\right)$ denote $\left(D^{4} \times T^{n-2}\right)_{1} \cup\left(D^{4} \times T^{n-2}\right)_{2}$. Standard methods involving cross-sections can be used to prove the following.

$$
\begin{aligned}
& \text { 3.1. LEMMA. } X\left(T_{1}, T_{2}, T_{3}\right) \cong S^{3} \times D^{2} \times T^{n-3} \text { iff } T^{n} \cong T_{1} \times T_{2} \times T_{3} \times \\
& T^{n-3} \text {. }
\end{aligned}
$$

The following is a refinement of a result mentioned earlier.

3.2. LEMMA. Suppose $T^{n}$ acts on a closed, connected, simply connected $(n+2)$-manifold $M$. If $\left(T_{1}, T_{2}\right)$ is an adjacent pair of $T^{1}$-stability groups then there exist $T^{1}$-stability groups $T_{3}, T_{4}, \ldots, T_{n}$ such that $T^{n} \simeq T_{1} \times T_{2} \times T_{3}$ $\times \cdots \times T_{n}$.

Proof. Let $k$ be the number of $T^{1}$-stability groups, where each such stability group $T_{i}$ is counted once for each component of $F\left(T_{i}, M\right)$. We fix $n$ and induct on $k$. If $k=n$, then $M \cong M_{n+2}$ and $T^{n}$ is isomorphic to the product of the $T^{1}$-stability groups so we are done. Therefore suppose the result is true for all $k<K$. Let $T^{n}$ act on $M$ with $K T^{1}$-stability groups $T_{1}, T_{2}, \ldots, T_{K}$ (not necessarily all distinct) where $\left(T_{K}, T_{1}\right)$ and $\left(T_{i}, T_{i+1}\right)$ are adjacent pairs for $1 \leqslant i<K$. The situation is illustrated in the following diagram. It suffices to show that there are $T^{1}$-stability groups $\left\{C_{3}, C_{4}, \ldots, C_{n}\right\}$ such that $T^{n} \cong T_{K} \times T_{1} \times C_{3} \times \cdots \times C_{n}$. We know from [11] that there is an adjacent triple $\left(T_{i}, T_{j}, T_{k}\right)$ such that $X\left(T_{i}, T_{j}, T_{k}\right) \cong$ $S^{3} \times D^{2} \times T^{n-3}$. Suppose $X\left(T_{i}, T_{j}, T_{k}\right) \approx S^{3} \times D^{2} \times T^{n-3}$ if $(i, j, k) \neq$ $(K, 1,2)$ or $(K-1, K, 1)$. Then we may equivariantly remove $X\left(T_{K}, T_{1}, T_{2}\right)$ or $X\left(T_{K-1}, T_{K}, T_{1}\right)$ and replace it with a copy of $D^{4} \times T^{n-2}$ where the action $\left(T^{n}, D^{4} \times T^{n-2}\right)$ has adjacent $T^{1}$-stability groups $\left(T_{K}, T_{2}\right)$ or $\left(T_{K-1}, T_{1}\right)$, respectively. The remaining $T^{1}$-stability groups will be $\left\{T_{2}, \ldots, T_{K}\right\}$ or $\left\{T_{1}, \ldots, T_{K-1}\right\}$ (listed as above). Continuing this process, the $T^{1}$-stability group eliminated will always be the first or last one listed. But $M_{n+2}$ must eventually be obtained by this process and, for an action $\left(T^{n}, M_{n+2}\right)$, each adjacent triple $\left(C_{i}, C_{j}, C_{k}\right)$ is such that $X\left(C_{i}, C_{j}, C_{k}\right) \approx S^{3}$ $\times D^{2} \times T^{n-3}$. Furthermore, at least one such triple will also be a triple for the action $\left(T^{n}, M\right)$ (other than $\left(T_{K-1}, T_{K}, T_{1}\right)$ or $\left(T_{K}, T_{1}, T_{2}\right)$ ). But this contradicts our assumption about such triples. Therefore there is a triple $(i, j, k) \neq(K, 1,2)$ or $(K-1, K, 1)$ such that $X\left(T_{i}, T_{j}, T_{k}\right)=S^{3} \times D^{2} \times$ $T^{n-3}$. Remove this and equivariantly replace it with a copy of $D^{4} \times T^{n-2}$ having $T^{1}$-stability groups $T_{i}$ and $T_{k}$. But $\left(T_{K}, T_{1}\right)$ will be an adjacent pair for the resulting action and the conclusion follows by induction. 


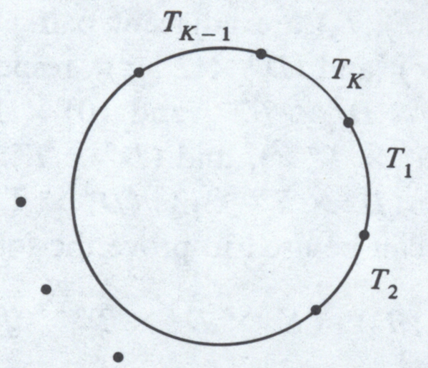

3.3. Lemma. Suppose $T^{n}$ acts on a closed, connected, simply connected $(n+2)$-manifold $M$. Let $U_{0} \cong D^{4} \times T^{n-2}$ be a tubular neighborhood of an orbit of type $T^{n-2}$. Then there is an $(n+2)$-cell $D^{n+2} \subset M$ such that $U_{0}$ is a $P L$ submanifold of $D^{n+2}$.

Proof. Suppose the action $\left(T^{n}, M\right)$ restricted to $U_{0}$ has adjacent $T^{1}$ stability groups $T_{1}$ and $T_{2}$. Then there are $T^{1}$-stability groups $T_{3}, T_{4}, \ldots, T_{n}$ for the action $\left(T^{n}, M\right)$ such that $T^{n} \cong T_{1} \times T_{2} \times T_{3} \times \cdots \times T_{n}$. Consider the following diagram. The action $\left(T^{n}, U_{0}\right)$ is the product of the linear action $\left(T_{1} \times T_{2}, D^{4}\right)$ and the free action $\left(T_{3} \times \cdots \times T_{n}, T^{n-2}\right)$ so $U_{0}$ can be triangulated so that there exists a PL submanifold $W \cong\left(T_{1} \times T_{2} \times I\right) \times$ $T^{n-2}$ of $\partial U_{0} \cong S^{3} \times T^{n-2}$. Let $\left(D^{2}\right)_{0}$ be a PL cell in $T_{1} \times T_{2}$, let

$$
V_{1}=T_{1} \times T_{2} \times I \times C\left(T_{3}\right) \times T_{4} \times \cdots \times T_{n} \cong T^{2} \times I \times D^{2} \times T^{n-3}
$$

and let $U_{1}=\left(D^{2}\right)_{0} \times I \times D^{2} \times T^{n-3}$. Form

$$
\begin{aligned}
Y_{1} & =U_{0} \cup_{\left(D^{2}\right)_{0} \times I \times T_{3} \times T^{n-3}} U_{1} \cong\left(D^{4} \times T^{n-2}\right) \cup_{D^{2} \times I \times T^{1} \times T^{n-3}} D^{5} \times T^{n-3} \\
& \cong\left(D^{4} \times T^{1} \cup_{D^{3} \times T^{1}} D^{5}\right) \times T^{n-3} \\
& \cong D^{5} \times T^{n-3} .
\end{aligned}
$$

Obviously $U_{0}$ is a PL submanifold of $Y_{1}$. Continuing in this manner for each stability group $T_{4}, \ldots, T_{n}$, we eventually obtain $Y_{n-2} \cong D^{n+2}$ with $U_{0}$ a PL submanifold of $D^{n+2}$.

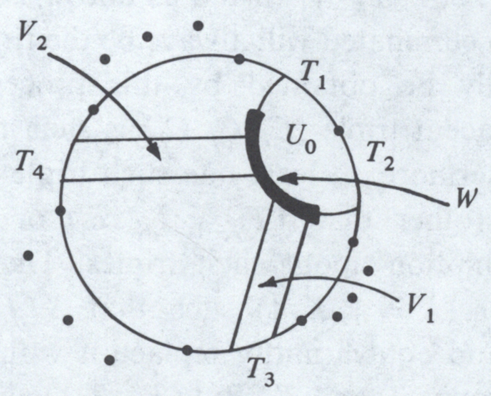

We now identify all the manifolds $M_{n+2}$. 


\subsection{THEOREM.}

$$
M_{n+2} \simeq \#_{j=1}^{n-3} j\left(\begin{array}{c}
n-2 \\
j+1
\end{array}\right) S^{2+j} \times S^{n-j}, \quad n>4
$$

Proof. We know that

$$
M_{6}=\#_{j=1}^{1} j\left(\begin{array}{c}
2 \\
j+1
\end{array}\right) S^{2+j} \times S^{4-j}=S^{3} \times S^{3}
$$

Now suppose that

$$
M_{n+1}=\#_{j=1}^{n-4} j\left(\begin{array}{c}
n-3 \\
j+1
\end{array}\right) S^{2+j} \times S^{n-j-1}
$$

$M_{n+2}$ can be obtained from $M_{n+1}$ in the following manner. Let $T^{n} \simeq T_{1}$ $\times \cdots \times T_{n}$ and consider the product actions $\left(T^{n-1} \times T_{n}, M_{n+1} \times S^{1}\right)$ and $\left(\left(T_{2} \times \cdots \times T_{n-2}\right) \times\left(T_{n-1} \times T_{n} \times T_{1}\right), T^{n-3} \times S^{5}\right)$. In the action $\left(T^{n-1}, M_{n+1}\right)$ let $\left(D^{4} \times T^{n-3}\right)_{1}$ be a tubular neighborhood of the orbit corresponding to the stability group $T_{n-1} \times T_{1}$. We then have $D^{4} \times T^{n-3} \times$ $S^{1}=\left(D^{4} \times T^{n-2}\right)_{1}$ embedded equivariantly in $M_{n+1} \times S^{1}$. Let $D^{4} \times T^{1}$ be a tubular neighborhood of the orbit corresponding to the stability group $T_{n-1} \times T_{1}$ in the action $\left(T_{n-1} \times T_{n} \times T_{1}, S^{5}\right)$. We then have $T^{n-3} \times D^{4} \times$ $T^{1}=\left(D^{4} \times T^{n-2}\right)_{2}$ equivariantly embedded in $T^{n-3} \times S^{5}$. It is not difficult to see that $M_{n+2} \cong\left(M_{n+1} \times S^{1}\right) \#_{T^{n-2}}\left(S^{5} \times T^{n-3}\right)$. The following diagram illustrates the situation. If we let $V=\#_{j=1}^{n-4} j\left(\begin{array}{c}n-3 \\ j+1\end{array}\right) S^{2+j} \times D^{n-j}$, then $M_{n+2}$ $\cong \partial\left(\left(V \times S^{1}\right) \#_{T^{n-2}}\left(D^{6} \times T^{n-3}\right)\right)$ so we compute $\left(V \times S^{1}\right) \#_{T^{n-2}}\left(D^{6} \times\right.$ $\left.T^{n-3}\right)$.

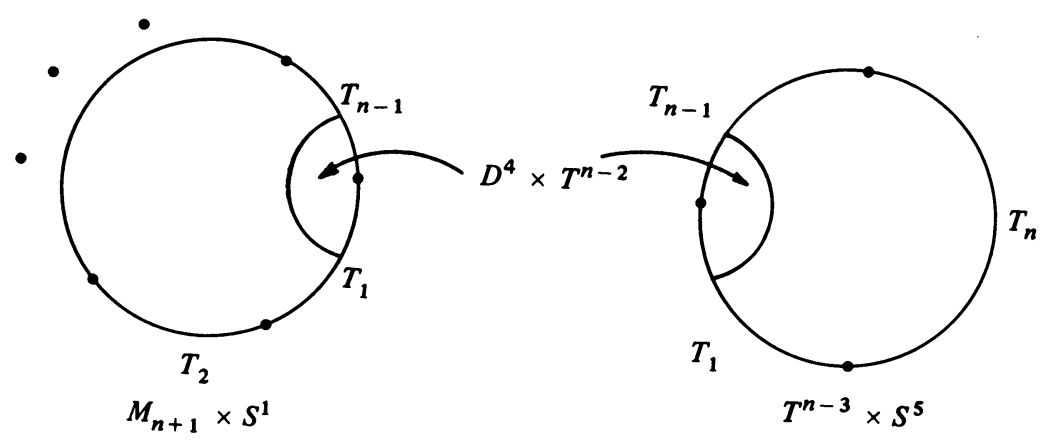

We know there exists a cell $\left(D^{n+1}\right)_{1} \subset M_{n+1} \cong \partial V$ such that $\left(D^{4} \times T^{n-3}\right)_{1}$ is a PL submanifold of $\left(D^{n+1}\right)_{1}$. Furthermore we know that with a specified triangulation $T$,

$$
V \cong\left(D^{n+2}\right)_{1} \cup_{X}\left(D^{n+2}\right)_{2}, \quad X=\#_{j=1}^{n-1} j\left(\begin{array}{c}
n-3 \\
j+1
\end{array}\right) S^{1+j} \times D^{n-j}
$$


where $\left(D^{n+2}\right)_{1},\left(D^{n+2}\right)_{2}$ and $X$ are PL submanifolds of $V$. We show that we may assume that $\left(D^{n+1}\right)_{1}$ and $\left(D^{4} \times T^{n-3}\right)_{1}$ are also PL submanifolds of $V$ and that $\left(D^{n+1}\right)_{1} \subset\left(D^{n+2}\right)_{1}$.

First, subdivide $V$ so that we have an $(n+1)$-simplex $P$ and a regular neighborhood $N$ of $P$ with $N \subset\left(D^{n+1}\right)_{1}^{\circ}$. Let $D_{1}$ and $D_{2}$ be connected polyhedra contained in $\partial V$ such that $\left(D^{n+1}\right)_{1} \subset D_{1}^{\circ} \subset D_{1} \subset D_{2}^{\circ}, D_{2} \neq \partial V$. Define a map $f: \partial V \rightarrow \mathbf{R}^{+}$such that

(1) $f^{-1}(0)=P$,

(2) $f$ is PL on $N$,

(3) $\frac{1}{4}<f(x)<\frac{1}{2}$ for $x \in D_{1}-N$,

(4) $f(x)>\frac{1}{2}$ for $x \in \partial V-D_{1}$,

(5) $f(x)=1$ for $x \in V-D_{2}^{\circ}$.

Approximate $f$ by a PL map $\bar{f}$ such that $f \cong \bar{f}$ on $N$ and $|\bar{f}(x)-f(x)|<\frac{1}{8}$ for all $x \in \partial V$. Then $\bar{f}^{-1}(0)=P$ and for some $\varepsilon, \frac{3}{4}<\varepsilon<1, U=\bar{f}^{-1}[0, \varepsilon]$ is a regular neighborhood of $P$. Thus $U \cong D^{n+1}$ and $\left(D^{n+1}\right)_{1} \subset U^{\circ}$.

If $X \cap U=\varnothing$ then we may assume that $U \subset\left(D^{n+2}\right)_{1}$. Otherwise choose a simplex $S \subset U$ and regular neighborhoods $N_{2}$ and $N_{3}$ of $S$ such that $N_{3} \subset N_{2}$ and $N_{2} \cap X=\varnothing$. Then there is an ambient isotopy of $V$ throwing $U$ onto $N_{2}$. Thus we may assume that the embedding $U \rightarrow \partial V$ is isotopic to an embedding that misses $X$ and we have $\left(D^{4} \times T^{n-3}\right)_{1} \subset\left(D^{n+1}\right), \subset U^{\circ} \subset \partial\left(D^{n+2}\right)_{1}$.

Let $\bar{U}$ be a PL $(n+2)$-cell in $V$ such that $\bar{U} \cap \partial V=U$. We have a triangulation of $\partial \bar{U}-\left(U^{\circ}\right)$ induced from $V$ and a triangulation on $\left(D^{n+1}\right)_{1}$ which makes $\left(D^{4} \times T^{n-3}\right)_{1}$ a PL submanifold of $\left(D^{n+1}\right)_{1}$. By Theorem 17 in [8], this triangulation of $\left(\partial \bar{U}-\left(U^{\circ}\right)\right) \cup\left(D^{n+1}\right)_{1}$ extends to all of $\bar{U}$. Thus $\left(D^{4} \times T^{n-3}\right)_{1} \subset\left(D^{n+1}\right)_{1} \subset\left(D^{n+2}\right)_{1} \subset V$ and all containments are PL.

To compute $\left(V \times S^{1}\right) \#_{T^{n-2}}\left(D^{6} \times T^{n-3}\right)$ we first find

$$
\begin{aligned}
\left(\left(D^{n+2}\right)_{1} \times S^{1}\right) \cup_{D^{4} \times T^{n-2}}\left(D^{6} \times T^{n-3}\right) & \\
& \simeq\left(\left(D^{n+2}\right)_{1} \times S^{1}\right) \#_{T^{n-2}}\left(D^{6} \times T^{n-3}\right) .
\end{aligned}
$$

This is $N_{1} \cong \#_{j-1}^{n-3}\left(\begin{array}{c}n-3 \\ j\end{array}\right) S^{2+j} \times D^{n-j+1}$.

We now need $\left(\left(D^{n+2}\right)_{2} \times S^{1}\right) \cup_{Y} N_{1}$ where

$$
Y \cong\left(\prod_{j=1}^{n-4} j\left(\begin{array}{c}
n-3 \\
j+1
\end{array}\right) S^{1+j} \times D^{n-j}\right) \times S^{1}=\bar{Y} \times S^{1}
$$

We have $\bar{Y} \subset D^{n+1} \subset \partial\left(D^{n+2}\right)_{1}$. (We can let $D^{n+1}=\partial\left(D^{n+2}\right)_{1}-\left(D^{n+1}\right)_{1}^{0}$.) Therefore $Y \subset D^{n+1} \times S^{1} \subset \partial N_{1}$. Since $S^{1}$ bounds in $\partial N_{1}$ we have $Y \subset$ $D^{n+1} \times S^{1} \subset D^{n+2} \subset \partial N_{1}$ and $Y \subset D^{n+1} \times S^{1} \times I \subset D^{n+2} \times I \subset N_{1}$. We may assume that $Y=\bar{Y} \times S^{1}=\bar{Y} \times \partial D^{2} \subset D^{n+1} \times D^{2} \subset N_{1}$. It follows that $\left(\left(D^{n+2}\right)_{2} \times S^{1}\right) \cup_{Y} N_{1} \cong N_{1} \# N_{2}$ where $N_{2}=\left(D^{n+1} \times D^{2}\right)$ $U_{Y}\left(\left(D^{n+2}\right)_{2} \times S^{1}\right)$. 
As in the proof of 2.3 we break $\left(D^{n+2}\right)_{2}$ up into $(n+2)$-cells $C_{k}$, one cell for each summand $S^{k} \times D^{n-k+1}$ of $\bar{Y}$ with $\bar{Y} \cap C_{k}=S^{k} \times D^{n-k+1}$. Then $\left(D^{n+2}\right)_{2} \times S^{1}$ is obtained by joining the $C_{k} \times S^{1}$ together along $D^{n+1} \times S^{1}$. Break $D^{n+1}$ up into $(n+1)$-cells $\bar{C}_{k}$ in a similar manner. We can form $N_{2}$ by first forming $\left(\bar{C}_{k} \times D^{2}\right) \#_{S^{k} \times S^{1}}\left(C_{k} \times S^{1}\right)$ for each $k$ and then joining these together along copies of $\left(D^{n} \times D^{2}\right) \cup_{D^{n} \times S^{1}} D^{n+1} \times S^{1} \simeq D^{n+2}$. Thus $N_{2}$ is the connected sum of all the $\left(\bar{C}_{k} \times D^{2}\right) \#_{s^{k} \times s^{1}}\left(C_{k} \times S^{1}\right)$. But

$$
\begin{aligned}
\left(\bar{C}_{k} \times D^{2}\right) & \#{ }_{S^{k} \times S^{1}}\left(C_{k} \times S^{1}\right) \\
& \simeq S^{k+1} \times D^{n-k+2} \# S^{k+2} \times D^{n-k+1} .
\end{aligned}
$$

Thus

$$
\begin{aligned}
& N_{2} \simeq\left(\#_{j=1}^{n-4} j\left(\begin{array}{l}
n-3 \\
j+1
\end{array}\right) S^{2+j} \times D^{n-j+1}\right) \\
& \#\left(\prod_{j=1}^{n-4} j\left(\begin{array}{l}
n-3 \\
j+1
\end{array}\right) S^{3+j} \times D^{n-j}\right)
\end{aligned}
$$

and

$$
\begin{aligned}
& \left(V \times S^{1}\right) \#_{T^{n-2}}\left(D^{6} \times T^{n-3}\right)=N_{1} \# N_{2} \\
& \simeq\left(\prod_{j=1}^{n-3}\left(\begin{array}{c}
n-3 \\
j
\end{array}\right) S^{2+j} \times D^{n-j+1}\right) \#\left(\begin{array}{c}
n-4 \\
\#=1
\end{array} j\left(\begin{array}{c}
n-3 \\
j+1
\end{array}\right) S^{2+j} \times D^{n-j+1}\right) \\
& \#\left(\prod_{j=1}^{n-4} j\left(\begin{array}{c}
n-3 \\
j+1
\end{array}\right) S^{3+j} \times D^{n-j}\right) \\
& =\left(\begin{array}{c}
n-3 \\
1
\end{array}\right) S^{3} \times D^{n} \#\left({\underset{j=2}{n-4}\left(\begin{array}{c}
n-3 \\
j
\end{array}\right) S^{2+j} \times D^{n-j+1}}_{j}\right) \\
& \#\left(\begin{array}{l}
n-3 \\
n-3
\end{array}\right) S^{n-1} \times D^{4} \\
& \#\left(\begin{array}{c}
n-3 \\
2
\end{array}\right) S^{3} \times D^{n} \#\left(\prod_{j=2}^{n-4} j\left(\begin{array}{c}
n-3 \\
j+1
\end{array}\right) S^{2+j} \times D^{n-j+1}\right) \\
& \#\left(\prod_{j=2}^{n-4}(j-1)\left(\begin{array}{c}
n-3 \\
j
\end{array}\right) S^{2+j} \times D^{n-j+1}\right) \\
& \#(n-4)\left(\begin{array}{l}
n-3 \\
n-3
\end{array}\right) S^{n-1} \times D^{4}
\end{aligned}
$$




$$
\begin{aligned}
& =\left(\begin{array}{c}
n-2 \\
2
\end{array}\right) S^{3} \times D^{n} \#\left(\begin{array} { c } 
{ n - 4 } \\
{ j = 2 }
\end{array} \left(\left(\begin{array}{c}
n-3 \\
j
\end{array}\right)+j\left(\begin{array}{c}
n-3 \\
j+1
\end{array}\right)\right.\right. \\
& \left.\left.+(j-1)\left(\begin{array}{c}
n-3 \\
j
\end{array}\right)\right) S^{2+j} \times D^{n-j+1}\right) \\
& \#(n-3)\left(\begin{array}{l}
n-3 \\
n-3
\end{array}\right) S^{n-1} \times D^{4} \\
& =\left(\begin{array}{c}
n-2 \\
2
\end{array}\right) S^{3} \times D^{n} \#\left(\begin{array}{c}
n-4 \\
j=2
\end{array} j\left(\begin{array}{c}
n-2 \\
j+1
\end{array}\right) S^{2+j} \times D^{n-j+1}\right) \\
& \quad \#(n-3)\left(\begin{array}{l}
n-2 \\
n-2
\end{array}\right) S^{n-1} \times D^{4} \\
& =\prod_{j=1}^{n-3} j\left(\begin{array}{l}
n-2 \\
j+1
\end{array}\right) S^{2+j} \times D^{n-j+1} .
\end{aligned}
$$

Computing the boundary of this proves the theorem.

The following lemma will be used to classify all $T^{n}$-actions on simply connected $(n+2)$-manifolds.

3.5. Lemma. Suppose $T^{n}$ acts on a closed, connected, simply connected $(n+2)$-manifold $M$ with $M=\partial N$. Let $D^{4} \times T^{n-2}$ be an invariant neighborhood of an orbit of type $T^{n-2}$ and let the embedding $\omega: D^{4} \times T^{n-2} \rightarrow S^{5} \times$ $T^{n-3}$ be the product of a standard embedding $T^{1} \times D^{4} \rightarrow S^{5}$ and the identity $T^{n-3} \rightarrow T^{n-3}$. With this embedding

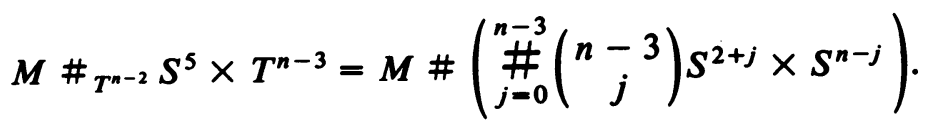

Proof. We first find $\bar{N}=N \#_{T^{n-2}} D^{6} \times T^{n-3}$. By 3.3 and 2.9,

$\bar{N} \simeq N \#\left(D^{n+3} \#_{T^{n-2}} D^{6} \times T^{n-3}\right) \simeq N \#\left(\prod_{j=0}^{n-3}\left(\begin{array}{c}n-3 \\ j\end{array}\right) S^{2+j} \times D^{n+1-j}\right)$.

Again, looking at the boundary proves the theorem.

As mentioned earlier, if $\left(T^{n}, M^{n+2}\right)$ is any action of $T^{n}$ on a simply connected $(n+2)$-manifold, then it can be obtained from an action $\left(T^{n}, M_{n+2}\right)$ by equivariantly replacing copies of $D^{4} \times T^{n-2}$ with copies of $S^{3} \times D^{2} \times T^{n-3}$. Since

$$
\begin{aligned}
S^{5} \times T^{n-3} & \cong\left(S^{3} \times D^{2} \cup D^{4} \times S^{1}\right) \times T^{n-3} \\
& \simeq S^{3} \times D^{2} \times T^{n-3} \cup D^{4} \times T^{n-2}
\end{aligned}
$$


we see that

$$
M^{n+2} \cong\left(\ldots\left(M_{n+2} \#_{T^{n-2}} S^{5} \times T^{n-3}\right) \# \ldots\right) \#_{T^{n-2}} S^{5} \times T^{n-3} .
$$

Since each time an $S^{5} \times T^{n-3}$ summand is added the number of orbits of type $T^{n-2}$ is increased by one, we have the following.

3.6. THEOREM. Suppose $T^{n}$ acts on a closed, compact, connected, simply connected $(n+2)$-manifold $M$ with $n+k$ orbits of type $T^{n-2}$. Then

$$
M=\#_{j=0}^{n-3}\left(j\left(\begin{array}{c}
n-2 \\
j+1
\end{array}\right)+k\left(\begin{array}{c}
n-3 \\
j
\end{array}\right)\right) S^{2+j} \times S^{n-j} .
$$

Proof. Fix $n$. By 3.4 the theorem is true for $k=0$, so suppose it is true for $K-1$ and let $T^{n}$ act on $M$ with $K$ orbits of type $T^{n-2}$. By induction, if

$$
\bar{M}=\#_{j=0}^{n-3}\left(j\left(\begin{array}{c}
n-2 \\
j+1
\end{array}\right)+(K-1)\left(\begin{array}{c}
n-3 \\
j
\end{array}\right)\right) S^{2+j} \times S^{n-j}
$$

then $M=\bar{M} \#_{T^{n-2}} S^{5} \times T^{n-3}$. Thus, by 3.5 ,

$$
\begin{aligned}
M & =\bar{M} \#\left(\prod_{j=0}^{n-3}\left(\begin{array}{c}
n-3 \\
j
\end{array}\right) S^{2+j} \times S^{n-j}\right) \\
& =\#_{j=0}^{n-3}\left(j\left(\begin{array}{c}
n-2 \\
j+1
\end{array}\right)+K\left(\begin{array}{c}
n-3 \\
j
\end{array}\right)\right) S^{2+j} \times S^{n-j}
\end{aligned}
$$

\section{BIBLIOGRAPHY}

1. D. Barden, Simply connected five-manifolds, Ann. of Math. (2) 82 (1965), 365-385. 1972.

2. G. E. Bredon, Introduction to compact transformation groups, Academic Press, New York,

3. R. Goldstein and L. Lininger, A classification of 6-manifolds with free $S^{1}$-actions, Proc. of the Second Conf. on Compact Transformation Groups, Univ. of Mass., 1971, Part 1, SpringerVerlag, Berlin and New York, 1972, pp. 316-323.

4. A. Haefliger, Knotted ( $4 k-1)$-spheres in 6k-space, Ann. of Math. (2) 75 (1962), 452-466.

5. J. F. P. Hudson, Piecewise linear topology, Benjamin, New York, 1969.

6. S. Kim, D. McGavran and J. Pak, Torus group actions on simply connected manifolds, Pacific J. Math. 53 (1974), 435-444.

7. S. Kim and J. Pak, Isotropy subgroups of torus $T^{n}$ actions on $(n+2)$-manifolds $M^{n+2}$, Michigan Math. J. 20 (1973), 353-359.

8. R. C. Kirby, Lectures on triangulations of manifolds (mimeographed), University of California at Los Angeles, 1969.

9. Dennis McGavran, $T^{3}$-actions on simply connected 6-manifolds. I, Trans. Amer. Math. Soc. 220 (1976), 59-85.

10.,$T^{3}$-actions on simply connected 6-manifolds. II, Indiana Univ. Math. J. 26 (1977), 125-136. 
11. __ $T^{n}$-actions on simply connected $(n+2)$-manifolds, Pacific J. Math. 71 (1977), 487-497.

12. J. Milnor and J. Stasheff, Characteristic classes, Ann. of Math. Studies, no. 76, Princeton Univ. Press, Princeton, N. J., 1974.

13. P. Orlik and F. Raymond, Actions of SO(2) on 3-manifolds, Proc. Conf. on Transformation Groups, New Orleans, 1967, Springer-Verlag, Berlin and New York, 1968, pp. 297-318.

14. Actions of the torus on 4-manifolds. I, Trans. Amer. Math. Soc. 152 (1970), 531-559.

15. J. Pak, Actions of the torus $T^{n}$ on $(n+1)$-manifolds $M^{n+1}$, Pacific J. Math. 44 (1973), 671-674.

16. F. Raymond, A classification of the actions of the circle on 3-manifolds, Trans. Amer. Math. Soc. 131 (1968), 51-78.

17. E. H. Spanier, Algebraic topology, McGraw-Hill, New York, 1966.

18. C. T. C. Wall, Classification problems in differential topology. V: On certain 6-manifolds, Invent. Math. 1 (1966), 355-374.

Department of Matheamatics, University of Connbcticut, Waterbury, Connecticut 06710 